\title{
Metabolomic Analysis of Natural Variation in Arabidopsis
}

\author{
Si Wu, Saleh Alseekh, Yariv Brotman, and Alisdair R. Fernie
}

\begin{abstract}
Methodological advances in coupled-mass spectrometry (gas chromatography and liquid chromatography; GC-MS and LC-MS) have rendered the profiling of highly complex plant extracts relatively facile and allowed that their high-throughput use aids the investigation of a range of biological questions. Among these is the elucidation of the genetic factors underlying metabolite abundance. For this purpose genomewide association studies (GWAS) are being widely adopted in Arabidopsis with the resultant quantitative trait loci being subjected to cross-validation by the use of recombinant inbred lines, introgression lines, and T-DNA insertional knockout lines.
\end{abstract}

Key words Metabolomics, QTL analysis, Genome-wide association mapping, Gas-chromatography mass-spectrometry, Liquid-chromatography mass-spectrometry

1 Introduction

The metabolites extant in the plant kingdom are extremely diverse; a commonly quoted estimate is that plants produce somewhere in the order of 200,000 unique chemical structures [1], with upward of 5000 being present in any given species [2]. Of these, some 1000 or so are primary metabolites which are essential for everyday cellular function and growth but the vast majority are specialized metabolites $[3,4]$ that are nonessential for growth and therefore not constitutively present in every cell type at every developmental stage. Recently, there has been an increasing use in analytical technologies such as metabolomics for comprehensive profiling of metabolites in biological samples in many different fields of biology including the characterization of natural variance in the plant model species Arabidopsis thaliana. Given the diversity of structural classes of metabolites, ranging from primary metabolites such as carbohydrates, amino acids, and organic acids to very complex secondary metabolites such as the Brassicacea-specific glucosinolates as well as phenolics, alkaloids, and terpenoids, there is no single methodology that can measure the complete metabolome 
in one step. It is, therefore, often necessary to combine different techniques to detect even a significant proportion of all metabolites within a complex mixture [5]. Both gas chromatography-mass spectrometry (GC-MS) and liquid chromatography-mass spectrometry (LC-MS) have been intensively used to profile broad natural variance in the form of recombinant inbred lines (RILs), introgression lines (ILs), and, more recently, genome-wide association mapping panels in order to enhance our understanding of the regulation of plant primary and secondary metabolism (see reviews $[6,7])$.

In all metabolomics applications, it is important to understand and control factors that contribute to sources of variation within the data sets. The variability between samples can arise from multiple sources including natural biological variation itself and that which occurs on sample collection and storage $[8,9]$. In addition, analytical variation caused by suboptimal performance of the apparatus used and instrument drift over time represent considerable hurdles in large-scale metabolomics studies [10] Batch-to-batch variation is the technical source of variation arising from the sum of both manual and robotic samples handling [11]. The presence of batch-to-batch variation makes it difficult to integrate data from independent batches of samples. This issue is particularly problematic when dealing with large number of samples such as is the case when analyzing structured plant populations.

To counter this, several normalization methods have been developed and suggested to overcome these issues and to minimize nonbiological variation [11-13]. For example, normalizations by a single or multiple internal or external standard compounds based on empirical rules, such as specific regions of retention time, have been used [14]. Similarly, isotope-labeled internal standard approaches were developed to monitor analytical error [15]. While there is no single best practice to conduct metabolomic studies, there are a number of pitfalls and known problems that need to be carefully avoided. Detailed guidelines and practice and normalization protocols have been published for this purpose [1618]. In this chapter, we describe a workflow for metabolomics GWAS studies in Arabidopsis including growth and harvesting, measurement of primary and secondary metabolites, association mapping, and cross- or functional validation of the identified associations.

Particularly the advent of genome-wide association studies, but also the use of mapping populations has greatly facilitated: (1) The association of genes to metabolic functions. (2) Both of structural and regulatory genes such as transcription factors which have been identified as controlling the accumulation of several specialized metabolite. (3) GWAS has become a key component of investigations into the interaction of metabolites with phenotypic traits such as growth [19] and the rates of photosynthesis and respiration 
[20, 21]. (4) Examples of associations between metabolites and biotic or abiotic stress tolerance have been uncovered which hopefully will pave the way toward the discipline of functional metabolomics [5] which will likely be founded using the model plant Arabidopsis thaliana.

\section{Materials}

\subsection{Plant material}

\subsection{GC-MS}

2.2.1 Sampling and Extraction

2.2.2 Derivatization
1. A large collection of Arabidopsis thaliana natural accessions such as the 314 natural accessions commonly referred to as the Arabidopsis HapMap collection ([22]; see Note 1). Alternatively, segregating populations resulting from biparental crosses such as introgression lines or recombinant inbred lines [23] or from multi-parental crosses following Magic like crossing schemes ([24]; see Note 2).

2. Suitable greenhouse or growth chamber conditions that are large enough to accommodate the populations in a manner that facilitates rapid harvest of samples from individual plants.

3. For validation experiments, knockout lines can be ordered from stock centers and genotyped as described in [25].

1. Argon.

2. Centrifuge (capable of $3700 \mathrm{~g}$ ), (e.g., Allegra ${ }^{\circledR} \mathrm{x}-15 \mathrm{R}$, Beckman Coulter).

3. Methanol gradient grade for liquid chromatography.

4. MilliQ water approx. $0.055 \mu \mathrm{S} / \mathrm{cm}$.

5. Oscillating ball mill MM200 (e.g., Retsch GmbH and Co.KG, Haan, Germany) or alternatively a pestle and mortar.

6. Ribitol, purity $\geq 99.0 \% ; 0.2 \mathrm{mg} / \mathrm{mL}$ in $\mathrm{dH}_{2} \mathrm{O}$.

7. Speed vacuum concentrator (e.g., SPDIllV-230, ThermoElectron Corporation, Langenselbold, Germany).

8. Schott glass AR-GLASS ${ }^{\circledR}$ culture tubes (soda-lime)

9. Thermoblock (capable of heating to up to $70{ }^{\circ} \mathrm{C}$ ).

10. Liquid nitrogen supply.

11. Vortex.

12. Scalpel blades, aluminum foil, 6-well plates, spatula, balance, microfuge tubes $(2 \mathrm{~mL})$.

1. Methoxyamine hydrochloride, purity $98 \%$. Store at room temperature under dry atmosphere.

2. $\mathrm{N}$-methyl- $\mathrm{N}$-trimethylsilyltrifluor(o)acetamide (MSTFA). MSTFA should be stored in opaque glass bottles under 
2.2.3 GC-TOF-MS

\subsection{LC-MS}

2.3.1 Sampling and Extraction

2.3.2 LC-MS Analysis nitrogen. Contact with water generates hydrogen fluoride gas which is highly toxic. Store at $4{ }^{\circ} \mathrm{C}($ see Note 3$)$.

3. Pyridine, analytic grade. Store at room temperature (see Note 3 ).

4. Retention time index standard mixture: fatty acids methyl esters (FAMES). All must be of standard grade for GC: Esters included are methylcaprylate, methyl pelargonate, methylcaprate, methyllaurate, methylmyristate, methylpalmitate, methylstearate, methyleicosanoate, methyldocosanoate, lignoceric acid methylester, methylhexacosanoate, methyloctacosanoate, and triacontanoic acid methylester. The esters are dissolved in $\mathrm{CHCl}_{3}$ at a final concentration of $0.8 \mu \mathrm{L} / \mathrm{mL}$ for liquid; $0.4 \mathrm{mg} / \mathrm{mL}$ for solid standards. Mix all well, aliquot into glass vials, and store at $-20{ }^{\circ} \mathrm{C}$.

5. $1.1 \mathrm{~mL}$ Screw Top Tapered Vial-Clear Gold Grade.

6. Shaker $(950 \mathrm{rpm})$.

1. Autosampler system.

2. Capillary column MDN-35, $30 \mathrm{~m} \times 0.32 \mathrm{~mm}, 0.25 \mu \mathrm{m}$ film thickness.

3. Conical single taper split/splitless liner.

4. Gas chromatograph, split and splitless injector with electronic pressure control up to 150 psi.

5. Helium 5.0 carrier gas.

6. Pegasus Tof mass analyzer and corresponding software.

1. Round-bottom-shaped $2 \mathrm{~mL}$ tube.

2. Round-bottom-shaped $1.5 \mathrm{~mL}$ tube.

3. Zirconia bead (3 $\mathrm{mm} \phi)$.

4. Internal standard.

5. Methanol (MeOH) ULC/MS grade. Caution: Methanol is toxic and should be handled under the fume hood.

6. Distilled water ULC/MS grade.

7. Extraction buffer (mixture of methanol:distilled water, $8: 2$ $(\mathrm{v} / \mathrm{v})$ containing internal standard).

1. LC-MS system with the operation software provided by MS manufacturer.

2. Glass vial.

3. Screw cap for glass vial.

4. Distilled water ULC/MS grade. 


\subsubsection{Data Analysis}

5. Acetonitrile (ACN) ULC/MS grade. Caution: Acetonitrile is toxic and should be handled under the fume hood.

6. Formic acid (HCOOH) ULC/MS grade. Caution: Formic acid is corrosive and should be handled under the fume hood.

1. Computer and Software for mass spectral data analysis (see [26]).

\section{Methods}

\subsection{Experimental Design}

3.2 Plant Material and Sampling
In order to correctly evaluate such large sample sets it is important to ensure that variables are manipulated under strictly controlled conditions while taking precise measurements. As such, the precision of an experiment critically depends on the size of the experiment and the homogeneity of experimental materials. In large genomics experiments, another critical step is to determine the number of lines and associated biological replicates. This is then followed by choosing the statistical approach to link genotype with phenotype. Here we neither focus on the choice of the number of lines nor the population structure needed to obtain a complete genotype-to-phenotype matrix in order to identify all possible QTL (see [27] for more details) but rather on how many biological replicates are required per line for acceptable statistical analysis and data normalization (see Note 4).

In QTL analysis the number of replicates profiled will have a major influence on the reliability and reproducibility of the data and consequently on the QTL mapping results. Therefore, the ability to make broad conclusions or identify causal genes using quantitative studies of metabolic variation is greatly influenced by the fact that metabolic abundances measured in these studies are highly dependent on the environmental, developmental, and genetic variations present within the experiment as well as the experimental error. Based on our own experience, it is recommended to use at least six independent biological replicates for each line (genotype) and many more control plants in a completely randomized design to overcome unavoidable effects associated with variation in microenvironmental factors such as light intensities, temperature, and air humidity. This should be planed carefully in advance and the population size and time needed for collecting the samples should also be taken into account in order to ensure that harvesting is carried out in as rapid and homogeneous a manner as possible.

1. In the case of introgression lines (ILs) a reasonable number of biological replicates is six independent plants (see Note 5)

2. Collect several different plant organs $(3-5)$ per biological replicate, pool them, and treat them as a single sample. 


\subsection{Sample \\ Processing and \\ Extraction}

3.5 Data Processing

\author{
3.6 Data \\ Normalization
}

\subsection{GWAS Mapping}

3. In the case of other population such as RILs, BILs, and GWAs, less replication is needed than in the ILs since in these populations genetic variance is represented in multiple lines, as opposed to a single line, within the population [28].

4. In order to minimize the variation introduced by sampling, there are several crucial points to take into consideration during harvest (see Note 6).

1. Harvest plant organs (e.g., leaves, flowers, or fruits) and freeze them immediately in liquid nitrogen to be stored at $-80^{\circ} \mathrm{C}$, or grind them immediately to a powder and start the extraction (see Note 7).

2. Before extraction quality control (QC, see Note 8) samples should prepared by pooling aliquots of individual study samples.

3. Distribute the QC samples across all machine-batches.

4. Extract, derivatize, and analyze aliquots thereof at the exact same time as the individual study samples [28].

1. In LC-MS extract directly introduced to the apparatus (see [29]).

2. In GC-MS based metabolomics, derivatization is needed which involves several general steps ([17, 18, 30, 31] for details).

3. We recommend to divide the samples in batches so that each batch contains 50-80 samples with ample QC samples distributed across the sequence run [28].

1. Once samples are analyzed, automatic data processing tools are required for peak picking and mass peak alignment (see Note 9).

1. Data normalization is a crucial step in any metabolomics study. First step is normalizing using an internal standard; this reduces the variation in sample extraction, and compared for machine drifts.

2. Further normalization to sample weight and QC samples is additionally useful ( see Note 10).

The basic principle of genome-wide association studies (GWAS), which were initially developed for use in medical genetics, is that the incidence of nucleotide polymorphisms is associated with the presence of variance, overcoming the limitations of using ILs and RILs (see Note 11). The step-by-step instructions for GWAS mapping are as follows: 
1. Obtain statistical significance levels for associations between traits and SNPs by applying mixed linear models (MLM), including both fixed and random effects. We usually take individuals as random effects, allowing MLM to incorporate information about relationships among individuals. The results of associations are usually demonstrated by Manhattan plots.

2. Identify candidate associations between traits and genes by comparing with other orthogonal approaches (e.g., correlation network analysis, eQTL) or with other genetic populations (RIL and IL). By doing this, we can find the overlapping associations by different methods or populations, increasing the chance of detecting true positive associations.

3. After narrowing down candidate associations, we can check SNP markers in the candidate genes in order to identify functional amino-acid substitution caused by polymorphism variants.

4. Perform linkage disequilibrium (LD) analysis of the mapped genomic region for the target trait, in order to reveal the relationships between the causal SNPs with the lead SNP.

5. Check whether the trait levels are significantly different between different informative haplotypes, potentially facilitating the identification of causal SNPs.

6. Generate a short list of candidate genes by incorporating biological knowledge.

7. Experimentally validate candidate genes by transgenic plants or in other genetic populations such as: (A) RIL mapping (see Note 12), (B) IL mapping (see Note 13), or (C) analysis of T-DNA insertional mutants (see Note 14).

In the following, we present several examples in order to demonstrate the analysis workflow of GWAS and its wide application in exploring causal metabolic genes, as well as the merit of combined use of network analysis with quantitative genetics:

A. Two loci ( $A O P$ and $M A M)$ predominantly contribute to the observed variations in aliphatic-glucosinolate formation in Arabidopsis [32, 33]. The AOP locus on chromosome 4 regulates side-chain modifications while the MAM locus on chromosome 5 controls chain elongation in the aliphaticglucosinolate biosynthesis pathway. For the 18 annotated aliphatic glucosinolates in Arabidopsis rosettes, subsequent GWAS results showed that almost all upstream and downstream aliphatic glucosinolates mapped to the MAM and/or AOP loci in control $\left(21^{\circ} \mathrm{C}\right.$ and $\left.150 \mu \mathrm{E} \mathrm{m}^{-2} \mathrm{~s}^{-1}\right)$ and stress conditions $\left(32{ }^{\circ} \mathrm{C}\right.$ and darkness) (Fig. 1, see Note 15), confirming previous findings $[32,34]$. In addition, the mapping results indicate that most MAM-regulated upstream 


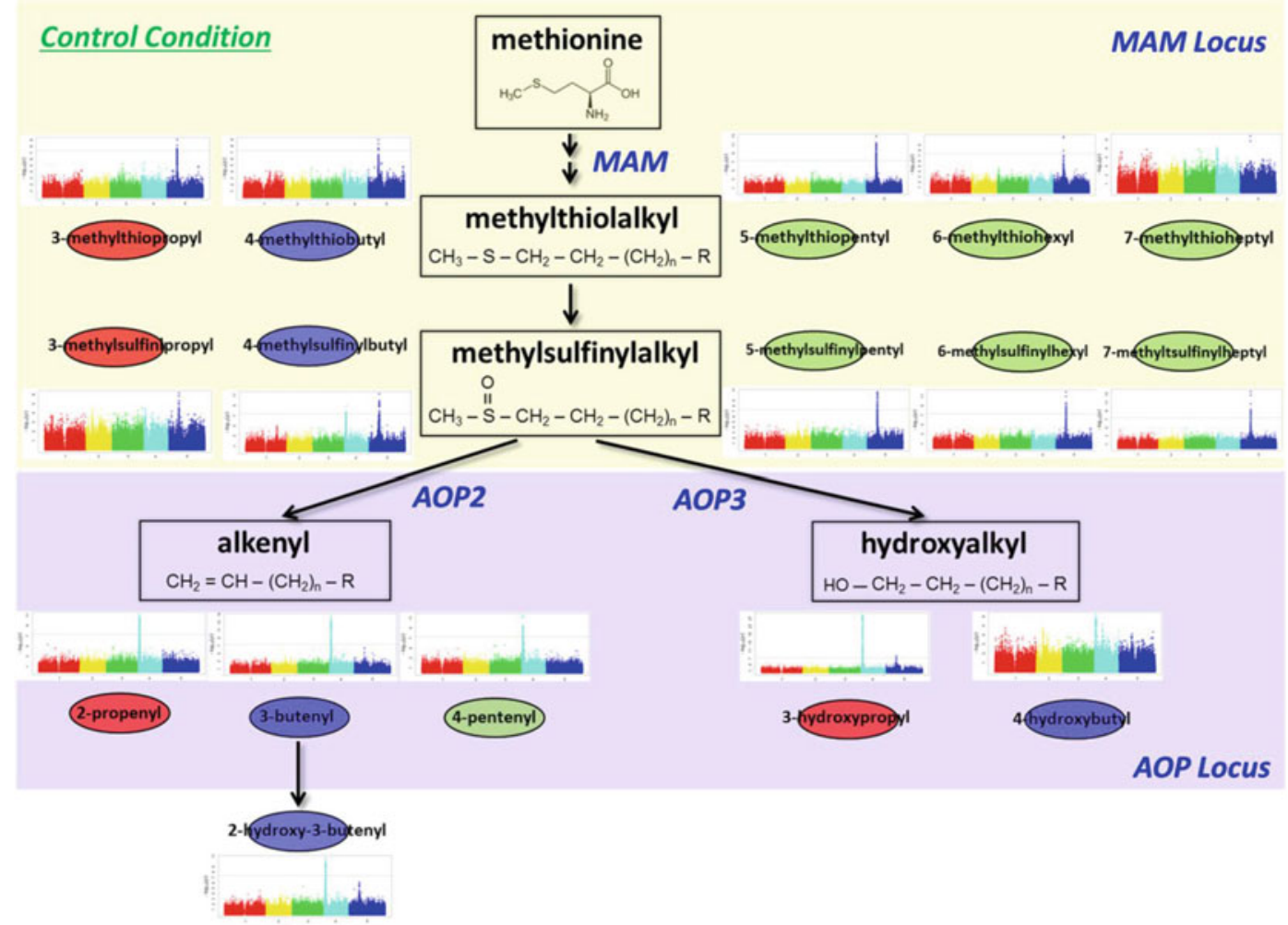

Fig. 1 The $M A M$ and $A O P$ loci regulate chain elongation and side-chain modification in aliphatic-glucosinolate biosynthesis pathway in the control condition (see Note 10)

metabolites (methylthiolalkyl and methylsulfinylalkyl glucosinolates) do not map to the $A O P$ locus while some $A O P$ regulated downstream metabolites (alkenyl and hydroxyalkyl glucosinolates) map to both the MAM and AOP loci (Fig. 1, see Note 15), which confirms the well-characterized aliphaticglucosinolate pathway in which $A O P$ acts downstream of $M A M$.

B. A strong link between an unknown metabolite trait $(\mathrm{m} / \mathrm{z}$ 256.0810 , retention time $=1.05$ ) and the candidate gene GCl (guanylyl cyclase 1 [35], AT5G05930) was supported by both GWAS (Fig. 2a, see Note 13) and network analysis in $21{ }^{\circ} \mathrm{C}$ and darkness (21-D) and $32{ }^{\circ} \mathrm{C}$ and darkness (32-D) conditions (Fig. 2b, see Note 16). In GCl, two SNPs $(\mathrm{ml64251}, \mathrm{G} / \mathrm{T}$, lead SNP; ml64253, C/A) result in an altered protein amino acid sequence (Fig. 2c, see Note 16). Together with the results from linkage disequilibrium (LD) (Fig. 2d, see Note 16) and haplotype (Fig. 2e, see Note 16) analyses, this finding suggests that these polymorphic variants are likely to constitute the functional variation underlying this association. Isotope-labeling results suggested the 
a

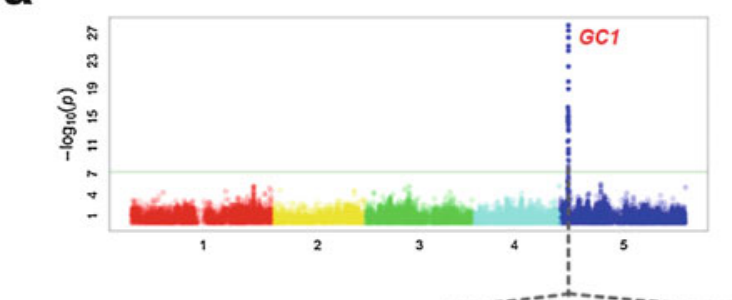

C

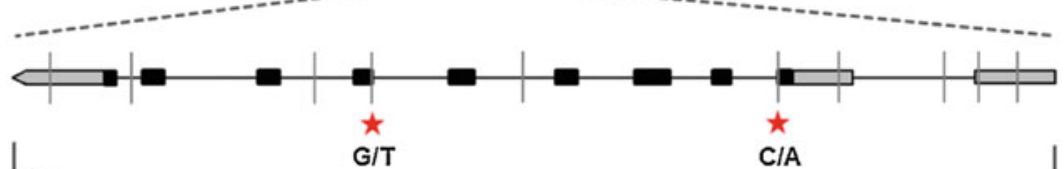

d

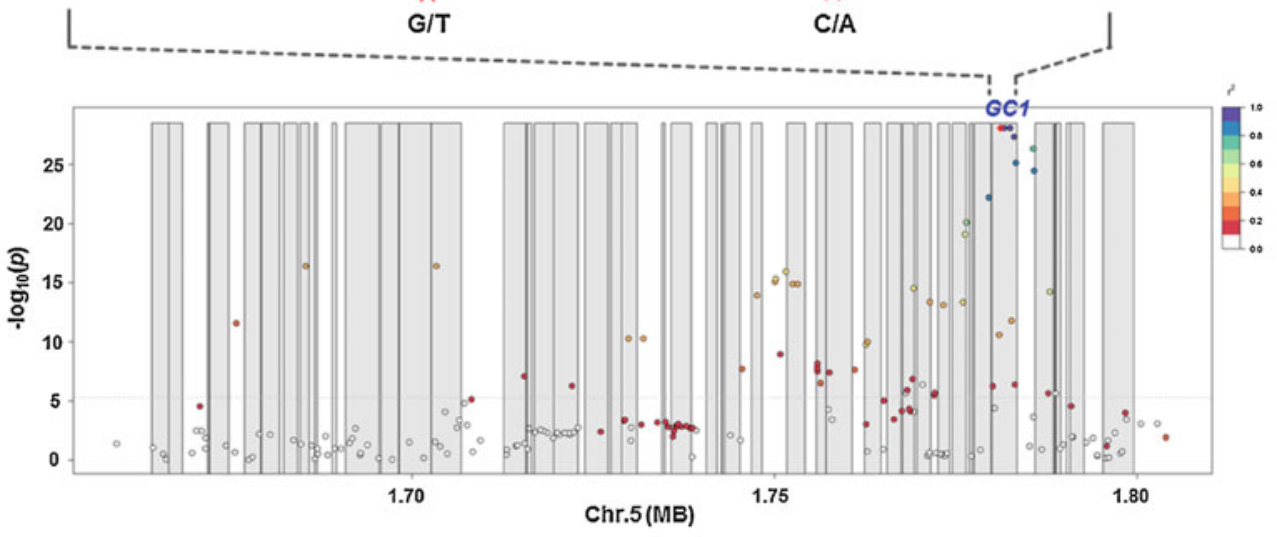

e

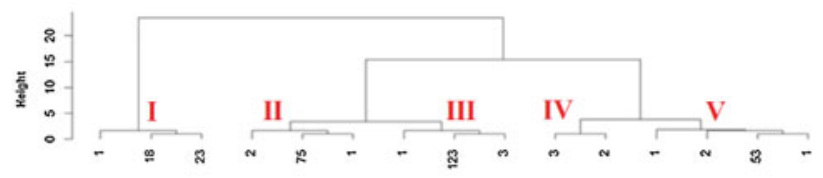

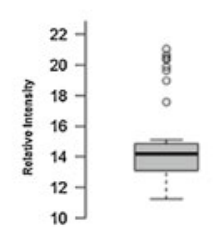

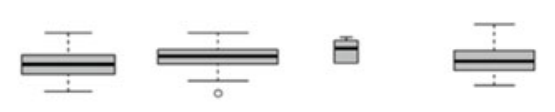

ANOVA: $p$-value $=1.72 \mathrm{e}-52$ (Bonferroni corrected)

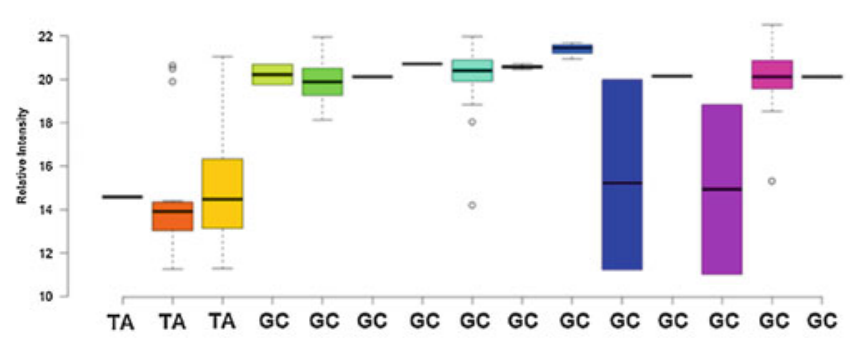

f
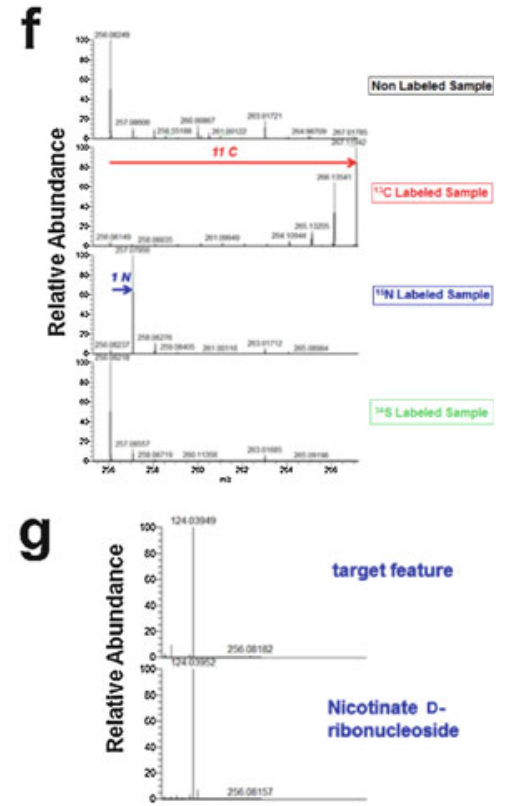

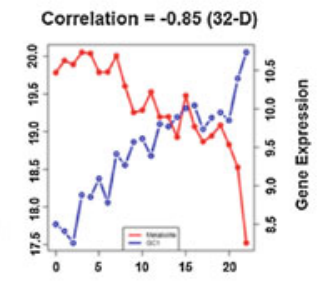

b correlation $=-0.65(21-D)$

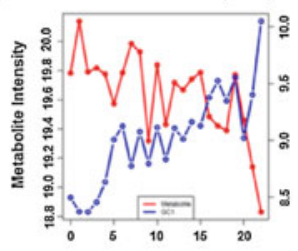


possible chemical formula $\mathrm{C}_{11} \mathrm{H}_{13} \mathrm{NO}_{6}$ (Fig. 2f, see Note 16). Assaying the standard compound nicotinate D-ribonucleoside revealed that it shares the same MS/MS fragmentation pattern as our metabolite (Fig. $2 \mathrm{~g}$, see Note 16), albeit with a slight retention-time shift, suggesting that our metabolite is structurally highly similar to nicotinate $d$-ribonucleoside.

C. A significant association ( $\mathrm{LOD}=5.46$ ), only appearing in the control-condition GWAS, was detected between gamma-Lglutamyl-L-cysteine and a locus on chromosome 5 (Fig. 3a, see Note 17) harboring two candidate genes: CYDS2 (cysteine synthase D2, AT5G28020) and DESI (L-cysteine desulfhydrase 1, AT5G28030). Both correlations between the metabolite feature and the two genes were supported by network analysis in darkness-related conditions (Fig. 3b, see Notes 17 and 18). We confirmed the metabolites as gamma-L-glutamyl-L-cysteine using isotope labeling (Fig. 3c, see Note 17) and MS/MS fragmentation analysis with a standard (Fig. 3d, see Note 14). DESI was reported to catalyze the desulfuration of cysteine to sulfide [36], whereas the function of CYSD2 remains elusive to date. Our results provide genetic evidence that the associations between gamma-L-glutamyl-L-cysteine and CYDS2 and DES1 are involved in cysteine metabolism (Fig. 3e, see Note 17).

\section{Notes}

1. Arabidopsis Hapmap population is a collection of $350 \mathrm{~A}$. thatiana accessions. The population was developed from a global collection of 5810 accessions [19]. This population has been genotyped for 248,584 bi-allelic single-nucleotide polymorphisms (SNPs). The SNPs were reduced to a set of 214,051 SNPs. For GWA analysis, SNPs with a minor allele frequency (MAF) higher than 0.05 were used for mapping (Yarive, $\mathrm{Si}$ ).

2. Segregating populations, such as recombinant inbred lines (RIL) and introgression lines (IL), are obtained from a cross (F1) of two parental accessions, through repeated selfing (RIL) or backcrossing of the initial hybrid with one of its parents (IL) followed by a selfing until a homozygous state is reached. Alternate alleles at loci in homozygous lines with distinct genetic basis in such structured populations potentially influence the trait of interest and allow to map the genomic loci responsible for the observed intra- or interspecific variation

3. Toxic chemicals should be handled in a fume hood with gloves.

4. In order to minimize the technical sources of variation, several samples are taken per plant, in addition to multiple independent replicates per parental genotypes; all the samples are 
a

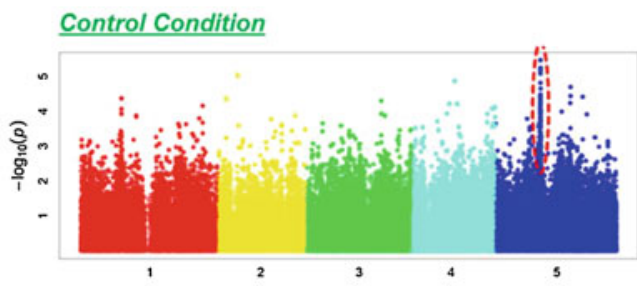

b
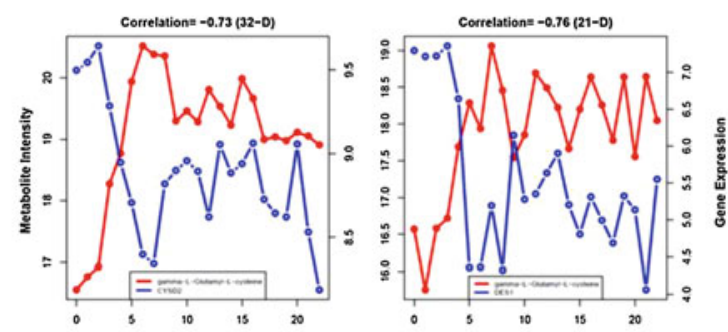

d

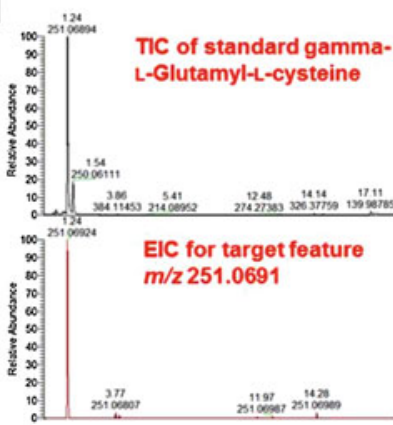

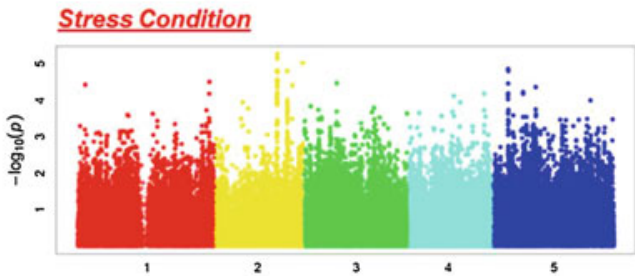

C

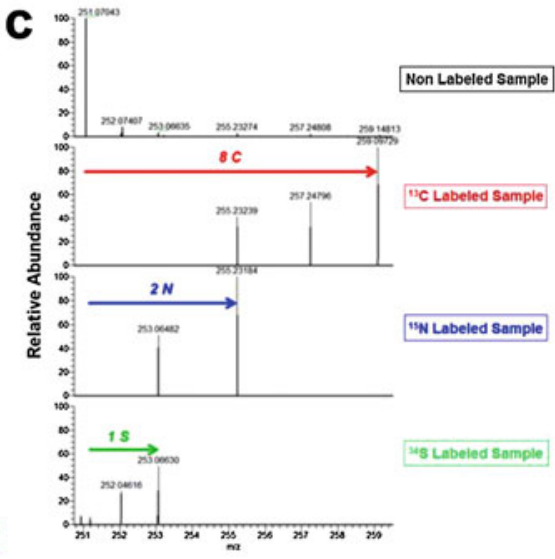

e

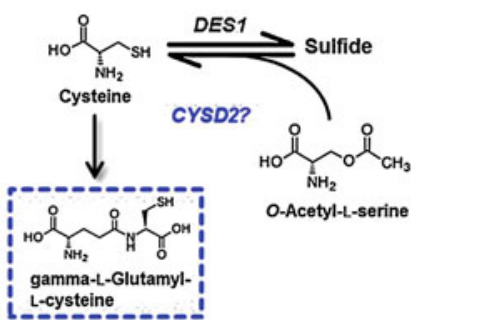

Fig. 3 Functional identification of the candidate associations between gamma-L-glutamyl-L-cysteine and a locus on chromosome 5 harboring CYDS2 and DES1 (see Note 13). (a) Manhattan plots for the metabolite trait gamma-L-glutamyl-L-cysteine in the control and stress conditions; (b) gamma-L-glutamyl-L-cysteine exhibits high negative correlations with both candidate genes (DES1 and CYSD2); (c) isotope labeling experiment assists annotation of chemical formula; (d) Annotation validation for gamma-L-glutamyl-L-cysteine by comparing MS/MS fragmentation pattern with chemical standard; (e) The biochemical pathway that the candidate associations are involved in

independently analyzed via metabolomics. The analysis of variance from this experiment will allow to estimate the variation between the replicates of same genotype and variation coming from genotypes. The optimum result is that most of the variance is due to genetic factors with the rest of the error being split between within replicates from the same plant or replicates between plants of the same genotype.

5. Plant sampling (harvesting) is a crucial step in sample preparation for metabolomics, and much care needs to be taken here. As mentioned above, the total variation in the data set is a 
function of different sources of variation including variation introduced by differences in sample collection. Large-scale experiments with vast sample size and genotype number (e.g., ILs, RILs or GWAS) have the added complication that some individuals may differ somewhat in their developmental age. However, having a large number of biological replicates represents an essential means to minimize metabolite variation during sample preparation even if it cannot account for all sources of this variation.

6. In case the experiment is too large to allow harvesting in a single and relatively short time, it is essential to harvest control samples from each temporally separate harvest. Further, plant metabolomics experiment are generally performed at the organ levels, and recommend to harvest pooled samples (several fruit or leafs) per biological replicate. In addition, the age or developmental stage should be carefully considered according to standardized growth condition and phenology descriptors.

7. All samples for a given experiment should follow exactly the same procedure before grinding, during extraction, and analyzing. Sample grinding is usually required to optimize solvent extraction and additionally aids in homogenizing the sample material [37]. Many extraction protocols are available for plant metabolomics and have been discussed in detail before (for example, see Shimizu et al. [29] for LC-MS, and [38] for GC-MS.). However, there are some important points at which these protocols should be adapted when handling the large number of plant samples required for QTL analysis. First, quality control (QC) (see Note 7) is necessary throughout the entire sample preparation process, from the greenhouse to the sample storage location and through distribution to chemical analysis and data normalization to reduce the analytical errors.

8. The quality control (QC) samples should represent, qualitatively and quantitatively, the entire samples included in the study and should provide an average of all of the metabolomes measured in the study. This can be done by pooling aliquots of individual samples, either all or a subset representative for the study. The QC samples should be evenly distributed over all the batches and are extracted, derivatized, and analyzed at the same time as the individual study samples. The data from the QC samples is used to monitor drift, separate high- and low-quality data, equilibrate the analytical platform, correct for drift in the signal, and allow the integration of multiple analytical experiments. The data analysis technique such as principal component analysis can be used to quickly assess the reproducibility of the QC samples in an analytical run. The QC samples are used to determine the variance of a metabolite feature. 
9. In GC-MS, several tools, software, and data bases have been established and used for this purpose (for more details see [2628]. For details on data processing of LC-MS data see [28]. For both methods, manual checking of the peaks is strongly recommended. REFINER MS® 10.0 (GeneData, http://www. genedata.com) can be used to analyze Chromatograms from the UPLC-FT/MS. From the raw files, the molecular masses, retention time, and associated peak intensities for each sample are extracted. The chromatogram alignments are performed using a pairwise alignment-based tree using $\mathrm{m} / \mathrm{z}$ windows of five points and RT windows of five scans within a sliding frame of 200 scans. Resulting data matrices with peak ID, retention time, and peak intensities in each sample are generated.

10. The goal of metabolomics as a phenotyping platform depends on its ability to detect biologically related metabolite changes in complex biological samples. As with any high-throughput technology, systematic biases are often observed in LC-MS and GC-MS metabolomics data [16, 28, 39]. As the number of samples in the data set increases, there is a corresponding timedependent variation in the metabolite data. Removing platform-specific sources of variability such as systematic errors is hence one of the top priorities in metabolomics data preprocessing. However, metabolite diversity leads to different responses to variations in given experimental conditions, making normalization a very demanding task [40]. For the effective elimination of different sources of analytical variation, preprocessing steps should follow a specific sequence. Here the Quality Control (QC) samples are of prime importance, and these are best prepared by pooling equal volumes of material from all of the biological samples to be analyzed. Alternatively, a chemically defined mixture of authenticated reference compounds [41] that mimics the metabolic composition of the investigated biological material can be employed [28]. Both the synthetic mixtures and biological QC samples should then be subjected to the same sample extraction, instrumental analyses (ideally distributed across the analytical run), and data processing, thus providing quality checks for technical and analytical error, and quantitative calibration to eliminate batch effects for the final processed data. This normalization is a crucial step for minimizing the batch-to-batch data variability across extended periods.

11. GWAS enables the analysis of associations between hundreds of thousands of single-nucleotide polymorphisms (SNPs) and specific traits. First the DNA obtained from hundreds to thousands of natural genetic accessions, and the accessions are genotypes and the genetic variations (like SNPs) are determined across the accessions. The principle based on If certain 
SNPs are found significantly more frequently in group of genotypes (accessions) with a certain phenotype (trait) than in the general population, the mutations are said to be "associated" with the trait. The GWAS analysis, represented in a Manhattan plot with significance $(-\log 10(P$ value $)$ on the $y$-axis, and genomic position shown as chromosomes in the $\mathrm{x}$-axis. This approach has several major advantages over conventional QTL mapping. First, a much larger and more representative gene pool can be surveyed. Second, it consumes less time and expense than mapping studies and enables the mapping of many traits in one set of genotypes. Third, a much finer mapping resolution can be achieved, resulting in small confidence intervals of the detected loci compared to classical mapping, where the identified loci need to be fine-mapped. Finally, it has the potential not only to identify and map QTLs but also to identify the causal polymorphism within a gene that is responsible for the difference in two alternative phenotypes [39]. A major issue with association studies is, however, a high rate of false positives, with the main source of these being the linkage between causal and non-causal sites and the confounding effects of population structure $[40,41]$. A high rate of false negatives, wherein loci with previous experimental validation for specific traits are not found in GWAS, may also appear; this is due to epistasis and lack of natural variation $[34,35]$. A quantitative genetics approach combined with metabolomics, whereupon metabolite levels are regarded as traits, can help unravel the genetic architecture of metabolic networks, identifying enzymes and regulatory genes that take part in specific metabolic pathways. Network analysis based on multiple omics data, as part of a top-down, complexity-reduction approach, can lead to original hypothesis generation about metabolic pathway regulation. Based on first principles, the integration of these two orthogonal approaches can accelerate the discovery of novel genes involved in plant metabolism with increased statistical confidence.

12. Cross-validation using RIL mapping: Immortal mapping populations consisting of homozygous individual have also been much used to map loci for complex metabolic traits (cite references). Recombinant inbred lines (RILs) can be obtained relatively easily and are produced by successively selfing the progeny of individual F2 plants (single seed descent method), from which the F8 generation and onward are practically homozygous lines that will produce further progeny that is essentially identical to the previous generation. Such a population can also be produced by induced chromosomal doubling of haploids, such as for doubled haploids (DHs; [28]). However RILs are advantageous over DHs given they are normally 
characterized by a higher frequency of recombination within the population, resulting from the multiple meiotic events which occur during repeated selfing [42]. For cross-validation metabolomics results from this population and the subsequent QTL mapping should provide consistent results.

13. Cross-validation using IL mapping: Another type of immortal population are introgression lines (IL) which are obtained through repeated backcrossing and extensive genotyping. These can also be referred to as near isogenic lines (NILs; [43]), or backcross inbred lines (BILs; [44, 45])—although the latter are slightly different in nature. These lines contain a single or a small number of genomic introgression fragments from a donor parent into an otherwise homogeneous genetic background [28]. For cross-validation metabolomics results from this population and the subsequent QTL mapping should provide consistent results.

14. Validation using T-DNA insertional mutants: Arguably even more reliable than cross-validation is the analysis of T-DNA insertional mutants-although it is important to note that caution needs to be taken to ensure that the accession background of these collections needs to be researched carefully in order to ascertain if it is suitable to validate the metabolic QTL or not since in the instance that the gene is not expressed in the accession in question an insertional mutation in that genotype will not yield a phenotype. If this is not the case ideally two independent insertional mutants per candidate gene should be ordered and genotyped [25] before the level of the metabolites that the gene associates is tested via the same metabolic profiling method described above.

15. Two loci ( $A O P$ and $M A M$ ) predominantly contribute to the observed variations in aliphatic-glucosinolate formation in Arabidopsis $[32,33]$. The $A O P$ locus on chromosome 4 regulates side-chain modifications while the MAM locus on chromosome 5 controls chain elongation in the aliphatic-glucosinolate biosynthesis pathway. For the 18 annotated aliphatic glucosinolates, subsequent GWAS results showed that almost all upstream and downstream aliphatic glucosinolates mapped to the MAM and/or $A O P$ loci in control and stress conditions (Fig. 1), confirming previous findings $[32,34]$. In addition, the mapping results indicate that most MAM-regulated upstream metabolites (methylthiolalkyl and methylsulfinylalkyl glucosinolates) do not map to the AOP locus while some AOP-regulated downstream metabolites (alkenyl and hydroxyalkyl glucosinolates) map to both the MAM and $A O P$ loci (Fig. 1), which confirms the well-characterized aliphatic-glucosinolate pathway in which $A O P$ acts downstream of $M A M$. 
16. A strong link between an unknown metabolite trait $(\mathrm{m} / \mathrm{z}$ 256.0810, retention time $=1.05$ ) and the candidate gene GCl (guanylyl cyclase l [35], AT5G05930) was supported by both GWAS (Fig. 2a) and network analysis in 21-D and 32-D conditions (Fig. 2b). In GCl, two SNPs (ml6425l, $\mathrm{G} / \mathrm{T}$, lead SNP; ml64253, C/A) result in an altered protein amino acid sequence (Fig. 2c). Together with the results from linkage disequilibrium (LD) (Fig. 2d) and haplotype (Fig. 2e) analyses, this finding suggests that these polymorphic variants are likely to constitute the functional variation underlying this association. Isotope-labeling results suggested the possible chemical formula $\mathrm{C}_{11} \mathrm{H}_{13} \mathrm{NO}_{6}$ (Fig. 2f). Assaying the standard compound nicotinate $D$-ribonucleoside revealed that it shares the same MS/MS fragmentation pattern as our metabolite (Fig. 2g), albeit with a slight retention-time shift, suggesting that our metabolite is structurally highly similar to nicotinate D-ribonucleoside.

17. A significant association ( $\mathrm{LOD}=5.46$ ), only appearing in the control-condition GWAS, was detected between gamma-Lglutamyl-L-cysteine and a locus on chromosome 5 (Fig. 3a) harboring two candidate genes: CYDS2 (cysteine synthase D2, AT5G28020) and DESI ( $L$-cysteine desulfhydrase 1, AT5G28030). Both correlations between the metabolite feature and the two genes were supported by network analysis in darkness-related conditions (Fig. 3b). We confirmed the metabolites as gamma-L-glutamyl-L-cysteine using isotope labeling (Fig. 3c) and MS/MS fragmentation analysis with a standard (Fig. 3d). DESI was reported to catalyze the desulfuration of cysteine to sulfide [36], whereas the function of CYSD2 remains elusive to date. Our results provide genetic evidence that the associations between gamma-L-glutamyl-Lcysteine and CYDS2 and DES1 are involved in cysteine metabolism (Fig. 3e).

18. The integration of genetic mapping and network analysis at this stage still mainly focused on limited classes of metabolites in Arabidopsis [34, 46, 47] and crop species [19, 48, 49]. It indicates that this integrative strategy coupled with large-scale untargeted metabolomics remained yet to be widely and deeply exploited, which can provide more global insights into the metabolic landscape of plants. In addition, plants interact with their surrounding environments from time to time by their numerous metabolites. Given the fact that many secondary metabolites are "silent" under control conditions, imposition of stresses may assist in the elucidation of novel biochemical pathways in plants. Therefore, the investigations of GWAS and network analysis with environmental stressdriven perturbations of metabolic homeostasis would largely 
lead to insightful discoveries of novel gene-metabolite associations involved in plants' regulatory systems toward the changing environments.

\section{Acknowledgements}

This work was in part supported by the PlantaSYST project by the European Union's Horizon 2020 research and innovation programme (SGA-CSA No 664621 and No 739582 under FPA No. 664620).

\section{References}

1. Rai A, Saito K, Yamazaki M (2017) Integrated omics analysis of specialized metabolism in medicinal plants. Plant J 90(4):764-787

2. Fernie AR et al (2004) Metabolite profiling: from diagnostics to systems biology. Nat Rev Mol Cell Biol 5(9):763-769

3. Weng JK, Philippe RN, Noel JP (2012) The rise of chemodiversity in plants. Science 336 (6089):1667-1670

4. Fraenkel GS (1959) The raison d'etre of secondary plant substances; these odd chemicals arose as a means of protecting plants from insects and now guide insects to food. Science 129(3361):1466-1470

5. Alseekh S, Fernie AR (2018) Metabolomics 20 years on: what have we learned and what hurdles remain? Plant J 94(6):933-942

6. Fang C, Luo J (2019) Metabolic GWAS-based dissection of genetic bases underlying the diversity of plant metabolism. Plant J 97 (1):91-100

7. Luo J (2015) Metabolite-based genome-wide association studies in plants. Curr Opin Plant Biol 24:31-38

8. Biais B et al (2012) Precautions for harvest, sampling, storage, and transport of crop plant metabolomics samples. In: Hardy NW, Hall RD (eds) Plant metabolomics: methods and protocols. Humana Press, Totowa, pp 51-63

9. Gibon Y, Rolin D (2012) Aspects of experimental design for plant metabolomics experiments and guidelines for growth of plant material. Methods Mol Biol 860:13-30

10. Sysi-Aho $M$ et al (2007) Normalization method for metabolomics data using optimal selection of multiple internal standards. BMC Bioinform 8:93

11. van der Kloet FM et al (2009) Analytical error reduction using single point calibration for accurate and precise metabolomic phenotyping. J Proteome Res 8(11):5132-5141

12. van der Greef J et al (2007) The art and practice of systems biology in medicine: mapping patterns of relationships. J Proteome Res 6 (4):1540-1559

13. Dunn WB et al (2008) Metabolic profiling of serum using ultra performance liquid chromatography and the LTQ-Orbitrap mass spectrometry system. J Chromatogr B Anal Technol Biomed Life Sci 871(2):288-298

14. Bijlsma $S$ et al (2006) Large-scale human metabolomics studies: a strategy for data (pre-) processing and validation. Anal Chem 78 (2):567-574

15. Chen MJ et al (2014) A modified data normalization method for GC-MS-based metabolomics to minimize batch variation. Springerplus 3:439

16. Fiehn O et al (2001) Metabolite profiling for plant functional genomics (vol 187, pg 1157, 2000). Nat Biotechnol 19(2):173-173

17. Fiehn O et al (2000) Metabolite profiling for plant functional genomics. Nat Biotechnol 18 (11):1157-1161

18. Lisec J et al (2006) Gas chromatography mass spectrometry-based metabolite profiling in plants. Nat Protoc 1(1):387-396

19. Wen W et al (2015) Genetic determinants of the network of primary metabolism and their relationships to plant performance in a maize recombinant inbred line population. Plant Cell 27(7):1839-1856

20. Nunes-Nesi A et al (2019) Identification and characterization of metabolite quantitative trait loci in tomato leaves and comparison with those reported for fruits and seeds. Metabolomics 15(4):46

21. de Oliveira Silva FM et al (2018) The genetic architecture of photosynthesis and plant 
growth-related traits in tomato. Plant Cell Environ 4l(2):327-34l

22. Horton MW et al (2012) Genome-wide patterns of genetic variation in worldwide Arabidopsis thaliana accessions from the RegMap panel. Nat Genet 44(2):212-216

23. Keurentjes JJ et al (2007) Development of a near-isogenic line population of Arabidopsis thaliana and comparison of mapping power with a recombinant inbred line population. Genetics 175(2):891-905

24. Wei J, Xu S (2016) A random-model approach to QTL mapping in multiparent advanced generation intercross (MAGIC) populations. Genetics 202(2):471-486

25. Timm S, Modde K, Bauwe H (2017) Targeted isolation and characterization of T-DNA mutants defective in photorespiration. Methods Mol Biol 1653:105-124

26. de Souza LP et al (2017) From chromatogram to analyte to metabolite. How to pick horses for courses from the massive web resources for mass spectral plant metabolomics. Gigascience 6(7): $1-20$

27. Joseph B, Corwin JA, Kliebenstein DJ (2015) Genetic variation in the nuclear and organellar genomes modulates stochastic variation in the metabolome, growth, and defense. PLoS Genet 11(1):el004779

28. Alseekh S et al (2018) Guidelines for sample normalization to minimize batch variation for large-scale metabolic profiling of plant natural genetic variance. Methods Mol Biol 1778:33-46

29. Shimizu T et al (2018) Targeted LC-MS analysis for plant secondary metabolites. Methods Mol Biol 1778:171-181

30. Allwood JW et al (2011) Plant metabolomics and its potential for systems biology research: background concepts, technology, and methodology. In: Jameson D, Verma M, Westerhoff $\mathrm{HV}$ (eds) Methods in enzymology. Methods in systems biology, vol 500. pp 299-336

31. Kopka J et al (2004) Metabolite profiling in plant biology: platforms and destinations. Genome Biol 5(6):831-841

32. Keurentjes JJ et al (2006) The genetics of plant metabolism. Nat Genet 38(7):842-849

33. Kliebenstein DJ et al (2001) Genetic control of natural variation in Arabidopsis glucosinolate accumulation. Plant Physiol 126(2):811-825

34. Chan EK et al (2011) Combining genomewide association mapping and transcriptional networks to identify novel genes controlling glucosinolates in Arabidopsis thaliana. PLoS Biol 9(8):e1001125
35. Wong A, Gehring C (2013) Computational identification of candidate nucleotide cyclases in higher plants. Methods Mol Biol 1016:195-205

36. Yamaguchi Y et al (2000) Three Arabidopsis genes encoding proteins with differential activities for cysteine synthase and betacyanoalanine synthase. Plant Cell Physiol 41 (4):465-476

37. Markert B (1995) Sample preparation (cleaning, drying, homogenization) for trace element analysis in plant matrices. Sci Total Environ 176(1-3):45-61

38. Osorio S, Do PT, Fernie AR (2012) Profiling primary metabolites of tomato fruit with gas chromatography/mass spectrometry. In Hardy NW, Hall RD (eds) Plant metabolomics: methods and protocols. Humana Press, Totowa, pp 101-109

39. Sehgal D, Singh R, Rajpal VR (2016) Quantitative trait loci mapping in plants: concepts and approaches. In: Rajpal VR, Rao SR, Raina SN (eds) Molecular breeding for sustainable crop improvement, vol 2, pp 31-59

40. Platt A, Vilhjalmsson BJ, Nordborg M (2010) Conditions under which genome-wide association studies will be positively misleading. Genetics 186(3):1045-1052

41. Larsson SJ, Lipka AE, Buckler ES (2013) Lessons from dwarf8 on the strengths and weaknesses of structured association mapping. PLoS Genet 9(2):e1003246

42. Jansen RC (2004) Quantitative trait loci in inbred lines. In: Handbook of statistical genetics. Wiley

43. Monforte AJ, Tanksley SD (2000) Development of a set of near isogenic and backcross recombinant inbred lines containing most of the Lycopersicon hirsutum genome in a L-esculentum genetic background: a tool for gene mapping and gene discovery. Genome 43 (5):803-813

44. Jeuken MJW, Lindhout P (2004) The development of lettuce backcross inbred lines (BILs) for exploitation of the Lactuca saligna (wild lettuce) germplasm. Theor Appl Genet 109 (2):394-401

45. Blanco A, Simeone R, Gadaleta A (2006) Detection of QTLs for grain protein content in durum wheat (vol 112, pg 1195, 2006). Theor Appl Genet 113(3):563-565

46. Wu S et al (2016) Combined use of genomewide association data and correlation networks unravels key regulators of primary metabolism in Arabidopsis thaliana. PLoS Genet 12(10): el006363 
47. Toubiana D et al (2015) Combined correlation-based network and mQTL analyses efficiently identified loci for branched-chain amino acid, serine to threonine, and proline metabolism in tomato seeds. Plant J 81 (1):121-133

48. Alseekh $S$ et al (2015) Identification and mode of inheritance of quantitative trait loci for secondary metabolite abundance in tomato. Plant Cell 27(3):485-512

49. Wen $W$ et al (2014) Metabolome-based genome-wide association study of maize kernel leads to novel biochemical insights. Nat Commun 5:3438 